\title{
ANOSOV AUTOMORPHISMS ON CERTAIN CLASSES OF NILMANIFOLDS
}

\author{
MEERA G. MAINKAR \\ School of Mathematics, Tata Institute of Fundamental Research, \\ Homi Bhabha Road, Colaba, Mumbai 400 005, India \\ e-mail:meera@math.tifr.res.in
}

(Received 6 August, 2005; accepted 28 October, 2005)

\begin{abstract}
We give a necessary and sufficient condition for $k$-step nilmanifolds associated with graphs $(k \geq 3)$ to admit Anosov automorphisms. We also prove the nonexistence of Anosov automorphisms on certain classes of 2-step and 3-step nilmanifolds.
\end{abstract}

2000 Mathematics Subject Classification. Primary 37D20. Secondary 22E25.

1. Introduction. A well-known class of Anosov diffeomorphisms arises as follows. Let $N$ be a simply connected nilpotent Lie group and let $\Gamma$ be a lattice in $N$; namely $\Gamma$ is a discrete subgroup such that $\Gamma \backslash N$ is compact. If $\tau$ is a hyperbolic automorphism (see $\S 2$ for the definition) of $N$ such that $\tau(\Gamma)=\Gamma$, then we get a diffeomorphism $\bar{\tau}$ of $\Gamma \backslash N$, defined by $\bar{\tau}(\Gamma x)=\Gamma \tau(x)$, for all $x \in N$, which is an Anosov diffeomorphism of the compact nilmanifold $\Gamma \backslash N$. Anosov diffeomorphisms arising in this way are called Anosov automorphisms of nilmanifolds. Let $K$ be a finite group of automorphisms of $N$ and let $\Gamma$ be a torsion free discrete cocompact subgroup of $K \ltimes N$. The $\Gamma$-action on $N$ is given by $(\tau, x) . y=x \tau(y)$, where $\tau \in K$ and $x, y \in N$. Now consider the quotient space $\Gamma \backslash N$ under the action of $\Gamma$ on $N$. We call such a compact manifold $\Gamma \backslash N$ an infranilmanifold. If $f$ is a hyperbolic automorphism of $N$ such that $f$ normalises the subgroup $K$ in the group of automorphisms of $N$ and $f(\Gamma)=\Gamma$, then $f$ induces a diffeomorphism $\bar{f}$ of the infranilmanifold $\Gamma \backslash N$; we call such a $\bar{f}$ an Anosov automorphism of an infranilmanifold $\Gamma \backslash N$.

The only known examples of Anosov diffeomorphisms are on nilmanifolds and infranilmanifolds. It is conjectured that any Anosov diffeomorphism is topologically conjugate to an Anosov automorphism of an infranilmanifold. By a result of A. Manning [8] all Anosov diffeomorphisms on nilmanifolds are topologically conjugate to Anosov automorphisms. This highlights the question of classifying all compact nilmanifolds which admit Anosov automorphisms. Indeed it is easy to see that not all of them do. The first example (due to Borel) of a non-toral nilmanifold admitting an Anosov automorphism was described by S. Smale [10]. Later L. Auslander and J. Scheuneman [1] gave a class of nilmanifolds admitting Anosov automorphisms.

By a result of S. G. Dani [2] all nilmanifolds covered by free $k$-step nilpotent Lie groups on $n$ generators, with $k<n$, admit Anosov automorphisms. There have been other recent constructions of compact nilmanifolds with Anosov automorphisms. (See [3], [5], [6], [7] and other references therein.)

In this paper we associate a $k$-step nilmanifold $(k \geq 3)$ with each graph, and give a necessary and sufficient condition, in terms of the graph, for the nilmanifold to 
admit Anosov automorphisms. We also prove some results on nonexistence of Anosov automorphisms on certain 2-step and 3-step nilmanifolds.

2. Preliminaries. In this section we recall some definitions and preliminaries concerning nilpotent Lie groups and nilmanifolds. We also recall results concerning automorphisms of a 2-step nilmanifold associated with a graph. (See [3] for details.)

Let $N$ be a simply connected nilpotent Lie group and $\mathcal{N}$ the Lie algebra of $N$. Note that $\mathcal{N}$ is nilpotent. Let $\operatorname{Aut}(N)$ denote the group of Lie automorphisms of $N$. Let $\operatorname{Aut}(\mathcal{N})$ denote the group of Lie algebra automorphisms of $\mathcal{N}$. Now $\operatorname{Aut}(N)$ is isomorphic to the group $\operatorname{Aut}(\mathcal{N})$, the isomorphism being given by $\tau \mapsto d \tau$, where $d \tau$ is the differential of $\tau$. Let $\Gamma$ be a discrete subgroup of $N$ such that $\Gamma \backslash N$ admits a finite $N$-invariant Borel measure. We call such a subgroup a lattice in $N$. As $N$ is a nilpotent Lie group, a discrete subgroup $\Gamma$ is a lattice in $N$ if and only if $\Gamma \backslash N$ is compact. (See Theorem 2.1 in [9].)

A nilmanifold is a quotient $\Gamma \backslash N$, where $N$ is a simply connected nilpotent Lie group and $\Gamma$ is lattice in $N$. An automorphism $\sigma \in \operatorname{Aut}(\mathcal{N})$ is said to be hyperbolic if all of its eigenvalues are of modulus different from 1. An automorphism $\tau \in \operatorname{Aut}(N)$ is said to be hyperbolic if all eigenvalues of the differential $d \tau$ are of modulus different from 1.

Now we recall the construction of the 2-step nilmanifold associated with a given graph and some results about its automorphism group. (See [3] for details.)

Let $(S, E)$ be a finite simple graph, where $S$ is the set of vertices and $E$ is the set of edges. Let $V$ be a real vector space with $S$ as a basis. Let $W$ be the subspace of $\wedge^{2} V$ spanned by $\{\alpha \wedge \beta: \alpha, \beta \in S, \alpha \beta \in E\}$, where $\wedge^{2} V$ is the second exterior power of $V$. Let $\mathcal{N}=V \oplus W$. We define the Lie bracket operation [, ] on $\mathcal{N}$ as follows. $[]:, \mathcal{N} \times \mathcal{N} \rightarrow \mathcal{N}$ is defined to be the unique bilinear map satisfying the following conditions:

(i) for $\alpha, \beta \in S,[\alpha, \beta]=\alpha \wedge \beta$ if $\alpha \beta \in E$ and 0 otherwise;

(ii) $[\alpha, \beta \wedge \gamma]=0$ for all $\alpha, \beta, \gamma \in S$;

(iii) $[\alpha \wedge \beta, \gamma \wedge \delta]=0$ for all $\alpha, \beta, \gamma, \delta \in S$.

We call $\mathcal{N}$ (defined as above) the 2-step nilpotent Lie algebra associated to the graph $(S, E)$. Let $N$ be the simply connected Lie group with Lie algebra $\mathcal{N}$. Let $\Gamma$ be the subgroup of $N$ generated by $\exp (S)$, where $\exp$ denotes the exponential map. It can be seen that $\Gamma$ is a lattice in $N$. A nilmanifold $\Gamma \backslash N$ is called the 2-step nilmanifold associated with the graph $(S, E)$.

For any $\sigma \in S$ we define

$$
\Omega^{\prime}(\sigma)=\{\omega \in S: \sigma \omega \in E\} \quad \text { and } \quad \Omega(\sigma)=\Omega^{\prime}(\sigma) \cup\{\sigma\}
$$

Let $\sim$ be an equivalence relation on $S$ defined as follows: for $\alpha, \beta \in S, \alpha \sim \beta$ if either $\alpha=\beta$ or $\Omega^{\prime}(\alpha) \subset \Omega(\beta)$ and $\Omega^{\prime}(\beta) \subset \Omega(\alpha)$. (See [3] for details.) Let $\left\{S_{\lambda}\right\}_{\lambda \in \Lambda}$ denote the set of all equivalence classes in $S$ with respect to the equivalence relation $\sim$, where $\Lambda$ is an index set. $S_{\lambda},(\lambda \in \Lambda)$, are called the coherent components of $S$. For each $\lambda \in \Lambda$, let $V_{\lambda}$ denote the subspace of $V$ spanned by $S_{\lambda}$.

We recall some results. See [3].

THEOREM 2.1. Let $(S, E)$ be a finite graph and let $\mathcal{N}=V \oplus W$ be the 2-step nilpotent Lie algebra associated with $(S, E)$ (notation as above). Let $G$ denote the subgroup of 
$G L(V)$ consisting of all restrictions, $\tau \mid V$, such that $\tau \in \operatorname{Aut}(\mathcal{N})$ and $\tau(V)=V$. Then $G$ is a Lie subgroup of $G L(V)$ and the following conditions are satisfied.

(i) The connected component of the identity in $G$ that we denote by $G^{0}$ can be expressed as $\left(\prod_{\lambda \in \Lambda} G L^{+}\left(V_{\lambda}\right)\right) \cdot M$, where, for each $\lambda \in \Lambda, G L^{+}\left(V_{\lambda}\right)$ denotes the subgroup of $G L\left(V_{\lambda}\right)$ consisting of all the elements with positive determinant and $M$ is a closed connected nilpotent normal subgroup of $G$.

(ii) The elements of $\Lambda$ can be arranged as $\lambda_{1}, \ldots, \lambda_{k}$ so that for all $j=1, \ldots, k$, $\bigoplus_{i \leq j} V_{\lambda_{i}}$ is invariant under the action of $G^{0}$.

LEMMA 2.2. Let $\lambda_{1}, \ldots, \lambda_{k}$ be an enumeration of $\Lambda$ such that assertion (ii) of Theorem 2.1 holds. For each $j=1, \ldots$, k let $\mathcal{N}_{j}=\left(\oplus_{i \leq j} V_{\lambda_{i}}\right) \oplus W$; also let $\mathcal{N}_{0}=W$. Let $\tau$ be a Lie automorphism of $\mathcal{N}$ contained in the connected component of the identity in $\operatorname{Aut}(\mathcal{N})$. Then each $\mathcal{N}_{j}$ is invariant under the action of $\tau$. Let $\Phi$ be the (additive) subgroup of $\mathcal{N}$ generated by $S \cup\left\{\frac{1}{2}(\alpha \wedge \beta: \alpha, \beta \in S, \alpha \beta \in E\}\right.$. If $\tau(\Phi)=\Phi$ then, for all $j=1, \ldots, k$, the determinant of the action of $\tau$ on $\mathcal{N}_{j}$ is \pm 1 .

3. $k$-step nilmanifold associated with the graph. In this section we associate a $k$ step $(k \geq 3$ ) nilmanifold (i.e. covered by a $k$-step simply connected nilpotent Lie group) with every graph and we give a necessary and sufficient condition for such nilmanifolds to admit an Anosov automorphism.

Starting with a graph $(S, E)$ we define a $k$-step $(k \geq 3)$ nilpotent Lie algebra as follows. Let $(S, E)$ be a finite graph, where $S$ is the set of vertices and $E$ is the set of edges. Suppose that $\mathcal{N}$ denotes the 2-step nilpotent Lie algebra associated with $(S, E)$ (see $\S 2$ ); i.e. $\mathcal{N}=V \oplus W$, where $V$ is a vector space with $S$ as a basis and $W$ is the subspace of $\wedge^{2} V$ spanned by $\{\alpha \wedge \beta: \alpha, \beta \in S, \alpha \beta \in E\}$.

Let $\mathcal{N}_{k}(V)$ be a free $k$-step nilpotent Lie algebra on $V$. (See [1] for the definition.) We denote by $\mathcal{H}_{k}$ the $k$-step nilpotent Lie algebra $\mathcal{N}_{k}(V) / \mathcal{J}$, where $\mathcal{J}$ denotes an ideal of $\mathcal{N}_{k}(V)$ generated by all elements $[\alpha, \beta]$ such that $\alpha \beta$ is not an edge. Let $N_{K}$ be the simply connected nilpotent Lie group with Lie algebra $\mathcal{H}_{k}$. Suppose that $\Phi_{k}$ is the (additive) subgroup of $\mathcal{H}_{k}$ generated by the elements of the type $[\alpha,[\beta, \ldots]]$, where $\alpha, \beta, \ldots \in S$. Then there exists a $\mathbb{Z}$-subalgebra $\Phi_{k}^{0}$ of $\mathcal{H}_{k}$ that is contained in $\Phi_{k}$ such that $\Gamma_{k}=\exp \left(\Phi_{k}^{0}\right)$ is a subgroup of $N_{k}$, and $\tau\left(\Phi_{k}\right)=\Phi_{k}$ if and only if $\tau\left(\Phi_{k}^{0}\right)=\Phi_{k}^{0}$, for any automorphism $\tau$ of $\mathcal{H}_{k}$. (See $\S 2$ in [1].) We note that $\Gamma_{k}$ is a lattice in $N_{k}$. We call a nilmanifold $\Gamma_{k} \backslash N_{k}$ a $k$-step nilmanifold associated with the graph $(S, E)$.

4. Anosov automorphisms of $\Gamma_{k} \backslash N_{k},(k \geq 3)$. We give a necessary and sufficient condition for the nilmanifold $\Gamma_{k} \backslash N_{k}$ to admit an Anosov automorphism.

Notation 4.1. Suppose that $\mathcal{H}$ is a $k$-step nilpotent Lie algebra. For any subset $M$ of $\mathcal{H}$ we denote $[M, M]$ by $M^{1},[M,[M, M]]$ by $M^{2}$, and inductively we denote $\left[M, M^{n-1}\right]$ by $M^{n}$ for all $n$ such that $3 \leq n \leq k-1$.

Let $(S, E)$ be a graph and $\Gamma_{k} \backslash N_{k}$ a $k$-step nilmanifold $(k \geq 2)$ associated with $(S, E)$. We refer to $\S 2$ and $\S 3$ for the notation.

REMARK 4.2. We note that any automorphism of $\mathcal{N}$ can be extended to an automorphism of $\mathcal{H}_{k}$. The automorphism group $\operatorname{Aut}\left(\mathcal{H}_{k}\right)$ is the semidirect product of $\operatorname{Aut}(\mathcal{N})$ and a connected group. This can be seen by observing that $\operatorname{Aut}\left(\mathcal{H}_{k}\right)$ is a semidirect product of $\operatorname{Aut}\left(\mathcal{H}_{k} / \mathcal{H}_{k}^{k-1}\right)$ and $\operatorname{Hom}\left(V, \mathcal{H}_{k}^{k-1}\right)$; also $\operatorname{Aut}\left(\mathcal{H}_{k} / \mathcal{H}_{k}^{2}\right)$ is the same as $\operatorname{Aut}(\mathcal{N})$. 
THEOREM 4.3. $\Gamma_{k} \backslash N_{k}$ admits an Anosov automorphism if and only if the following conditions hold.

(i) For every $\lambda,\left|S_{\lambda}\right| \geq 2$;

(ii) If $\left|S_{\lambda}\right|=l$, with $2 \leq l \leq k$, and $\alpha, \beta \in S_{\lambda}$, then $\alpha \beta$ is not an edge.

Proof. Suppose that, for each $\lambda \in \Lambda$, (i) and (ii) hold. We shall prove that there exists a hyperbolic automorphism $\tau \in \operatorname{Aut}\left(\mathcal{H}_{k}\right)$ such that $\tau\left(\Phi_{k}\right)=\Phi_{k}$.

We have $V=\oplus_{\lambda \in \Lambda} V_{\lambda}$. (See $\S 2$ for the notation.) For each $\lambda \in \Lambda$, let $\Phi_{\lambda}$ be the subgroup of $V_{\lambda}$ generated by $S_{\lambda}$. There exists $g_{\lambda} \in G L\left(V_{\lambda}\right)$ such that $g_{\lambda}\left(\Phi_{\lambda}\right)=$ $\Phi_{\lambda}$ if and only if the matrix representing $g_{\lambda}$ with respect to the basis $S_{\lambda}$ belongs to $G L\left(d_{\lambda}, \mathbb{Z}\right)$, where $d_{\lambda}=\left|S_{\lambda}\right|$. For each $\lambda \in \Lambda$ there exists a matrix $A_{\lambda} \in G L\left(d_{\lambda}, \mathbb{Z}\right)$ with the eigenvalues $c_{1}, c_{2}, \ldots c_{d_{\lambda}}$ such that $\left|c_{i_{1}} c_{i_{2}} \cdots c_{i_{r}}\right| \neq 1$, for all $r$ with $1 \leq r \leq$ $\min \left(k, d_{\lambda}-1\right)$, and for all $i_{1}, i_{2}, \ldots, i_{r} \in\left\{1,2, \ldots, d_{\lambda}\right\}$. The existence of such elements can be proved by using a result of S. G. Dani. (See Corollary 4.7 in [4].) Let $g_{\lambda}$ denote the transformation from $G L\left(V_{\lambda}\right)$ whose matrix with respect to the basis $S_{\lambda}$ is $A_{\lambda}$. By the above observation $g_{\lambda}\left(\Phi_{\lambda}\right)=\Phi_{\lambda}$. We choose natural numbers $j_{\lambda}, \lambda \in \Lambda$, such that $\left|\prod_{\lambda \in \Omega}\left(c_{\lambda i_{1}} c_{\lambda i_{2}} \cdots c_{\lambda i_{n_{\lambda}}}\right)\right| \neq 1$, for all subsets $\Omega$ of $\Lambda$ such that $|\Omega| \geq 2$ and $2 \leq \sum_{\lambda \in \Omega} n_{\lambda} \leq k$, where $c_{\lambda i_{j}}$ 's are eigenvalues of $g_{\lambda}$. Let $g \in G L(V)$ be the element whose restriction to $V_{\lambda}$ is $g_{\lambda}^{j_{\lambda}}$, for each $\lambda \in \Lambda$.

There exists $\bar{\tau} \in \operatorname{Aut}(\mathcal{N})$ such that $g$ is the restriction of $\bar{\tau}$ to $V$. (See Theorem 2.1.) We know that $\bar{\tau}$, constructed as above, is a hyperbolic automorphism of $\mathcal{N}$. This can be seen from the proof of Theorem 1.1 in [3] and the hypothesis of the theorem. Let $\tau$ be an automorphism of $\mathcal{H}_{k}$ obtained by extending $\bar{\tau}$. We note that $\tau\left(\Phi_{k}\right)=\Phi_{k}$, by construction. We shall prove that $\tau$ is hyperbolic as a linear transformation. Suppose that if possible $\tau$ has an eigenvalue, say $c$, of absolute value 1 . Then $c$ must be an eigenvalue of the restriction of $\tau$ to $V^{n}$, where $3 \leq n \leq k$ (see Notation 4.1), since $\tau$ is hyperbolic on $\mathcal{N}$.

Now using the fact that $\tau\left(V_{\lambda}\right)=V_{\lambda}$, for all $\lambda \in \Lambda$ and recalling the construction of $g$, we see that there exists $\lambda \in \Lambda$ such that $\left|S_{\lambda}\right|=n$ and $V_{\lambda}^{n}$ is nonzero. (See Notation 4.1.) But by the condition in the hypothesis $\alpha \beta$ is not an edge, for all $\alpha, \beta \in S_{\lambda}$. Hence $[\alpha, \beta]=0$, for all $\alpha, \beta \in S_{\lambda}$. This contradiction shows that $\tau$ is hyperbolic. Hence $\Gamma_{k} \backslash N_{k}$ admits an Anosov automorphism.

Conversely suppose that $\Gamma_{k} \backslash N_{k}$ admits an Anosov automorphism. Hence there exists $\tau \in \operatorname{Aut}\left(\mathcal{H}_{k}\right)$ such that $\tau\left(\Phi_{k}\right)=\Phi_{k}$ and $\tau$ is a hyperbolic linear transformation. Let $\bar{\tau} \in \operatorname{Aut}(\mathcal{N})$ denote an automorphism of $\mathcal{N}$ induced by $\tau$. We can assume that $\bar{\tau}(\Phi)=\Phi$, where $\Phi$ is the subgroup of $\mathcal{N}$ (with respect to addition) generated by the subset $S \cup\left\{\frac{1}{2}(\alpha \wedge \beta): \alpha, \beta \in S, \alpha \beta \in E\right\}$. As $\bar{\tau}$ is a hyperbolic linear transformation, $\left|S_{\lambda}\right| \geq 2$ for every $\lambda$, and if $\left|S_{\lambda}\right|=2$ then $\alpha \beta$ is not an edge for $\alpha \beta \in S_{\lambda}$. (See Theorem 1.1 in [3].) We may assume that $\bar{\tau}$ is contained in the connected component of the identity in $\operatorname{Aut}(\mathcal{N})$. (See Remark 4.2). Let $G$ denote the subgroup of $G L(V)$ consisting of all restrictions $\tau \mid V$ such that $\tau \in \operatorname{Aut}(\mathcal{N})$ and $\tau(V)=V$. We write the elements of $\Lambda$ as $\lambda_{1}, \lambda_{2}, \ldots, \lambda_{m}$ such that, for all $j=1, \ldots, m, \bigoplus_{i \leq j} V_{\lambda_{i}}$ is invariant under the action of $G^{0}$, where $G^{0}$ is the connected component of the identity in $G$. (See Theorem 2.1.) Now suppose that there exists $\lambda \in \Lambda$ such that $\left|S_{\lambda}\right|=l$, where $3 \leq l \leq k$ and $\alpha \beta \in E$, for all $\alpha, \beta \in S_{\lambda}$. Let $j$, with $1 \leq j \leq m$, be such that $\lambda=\lambda_{j}$. Consider the induced action of $\bar{\tau}$ on $\mathcal{N}_{j} / \mathcal{N}_{j-1}$, where $\mathcal{N}_{j}=\left(\oplus_{i \leq j} V_{\lambda_{i}}\right) \oplus W$. We note that each $\mathcal{N}_{j}$ is invariant under the action of $\bar{\tau}$. (See Lemma 2.2.) As the determinant of the induced action of $\bar{\tau}$ on $\mathcal{N}_{j} / \mathcal{N}_{j-1}$ is \pm 1 , the product of the eigenvalues $\theta_{1}, \theta_{2}, \ldots, \theta_{l}$ of the induced action is \pm 1 . Since the action is hyperbolic, at least two eigenvalues, say $\theta_{1}$ and $\theta_{2}$, are distinct. Hence 
there exist $v_{1}, v_{2}, \ldots, v_{l} \in V_{\lambda_{j}}^{\mathbb{C}}$ (the complexification of $V_{\lambda_{j}}$ ) such that $\bar{\tau}\left(v_{i}\right)=\theta_{i} v_{i}+x_{i}$, where $x_{i} \in \mathcal{N}_{j-1}^{\mathbb{C}}$, for all $1 \leq i \leq l$. We note that $v_{1}$ and $v_{2}$ are linearly independent, since $\theta_{1}$ and $\theta_{2}$ are distinct. We write $v_{i}=\sum_{\alpha \in S_{\lambda_{j}}} a_{\alpha}^{i} \alpha$, where $a_{\alpha}^{i} \in \mathbb{C}$ for all $\alpha \in S_{\lambda_{j}}$ and $1 \leq i \leq l$. As $v_{1}$ and $v_{2}$ are linearly independent and $\alpha \beta \in E$, for all $\alpha, \beta \in S_{\lambda_{j}}$, we have $\left[v_{1}, v_{2}\right] \neq 0$ in $\mathcal{N}^{\mathbb{C}}$. Hence $\left[v_{l},\left[\cdots,\left[v_{2}, v_{1}\right] \cdots\right]\right] \neq 0$ in $\mathcal{H}_{k}$. Let $x=\left[v_{l},\left[\cdots,\left[v_{2}, v_{1}\right] \cdots\right]\right]$. By considering the complexification of $\tau$ and $\bar{\tau}$ we have $\tau^{\mathbb{C}}(x)=\left(\prod_{i=1}^{l} \theta_{i}\right) x+y$, where $y$ belongs to the complexification of $\left[\mathcal{N}_{j},\left[\mathcal{N}_{j}, \cdots\left[\mathcal{N}_{j}, \mathcal{N}_{j-1}\right] \cdots\right]\right]_{(l-1) \text { times }}$, which we denote by $W^{\prime}$. We note that $\prod_{i=1}^{l} \theta_{i}= \pm 1$ and $x \notin W^{\prime}$. Hence we have an eigenvalue \pm 1 for the induced action of $\tau$ on $\left(\mathcal{N}_{j}^{l}\right)^{\mathbb{C}} / W^{\prime}$ which is a contradiction, since by assumption $\tau$ is hyperbolic. This shows that $\alpha \beta$ is not an edge for all $\alpha, \beta \in S_{\lambda}$, where $\left|S_{\lambda}\right|=l, 1 \leq$ $l \leq k$. This completes the proof of the theorem.

ExAmPLes 4. (i) Let $(S, E)$ be a complete graph; that is $\alpha \beta \in E$, for all $\alpha, \beta \in S$. Then the corresponding $k$-step nilmanifold admits an Anosov automorphism if and only if $|S|>k$.

(ii) Let $(S, E)$ be a cycle on 4 vertices. The corresponding $k$-step nilmanifold admits an Anosov automorphism for all $k \geq 2$. In particular, we get an example of 20-dimensional 3-step nilmanifold admitting an Anosov automorphism.

(iii) A complete bipartite graph $(S, E)$ is a graph in which $S$ is a disjoint union of two subsets $S_{1}$ and $S_{2}$, each containing at least two elements, and $E=\left\{\alpha \beta: \alpha \in S_{1}, \beta \in S_{2}\right\}$. In this case $S_{1}$ and $S_{2}$ are the coherent components. Hence the $k$-step nilmanifold associated with a complete bipartite graph admits an Anosov automorphism for all $k \geq 2$. In particular, if we choose $S_{1}$ and $S_{2}$ such that $\left|S_{1}\right|=m$ and $\left|S_{2}\right|=n$ we get an example of an $l$-dimensional 3-step nilmanifold admitting an Anosov automorphism, where

$$
l=m(n-1)^{2}-\frac{(n-2)(n-1) m}{2}+n(m-1)^{2}-\frac{(m-2)(m-1) n}{2}+2 m n .
$$

(iv) Let $(S, E)$ be a "magnet" graph with core $C$; i.e. $C$ is a subset of $S$ such that its complement in $S$ contains at least two elements and $E=\{\alpha \beta: \alpha \in C, \beta \in S, \alpha \neq \beta\}$. The $k$-step nilmanifold associated with $(S, E)$ admits an Anosov automorphism if and only if $k<|C|$.

5. Nonexistence of Anosov automorphisms on certain 2-step nilmanifolds. In this section we prove some results on nonexistence of Anosov automorphisms on certain nilmanifolds. Let $\mathcal{N}_{\mathbb{Q}}$ be the 2-step nilpotent Lie algebra over $\mathbb{Q}$, associated to the graph $(S, E)$. Let $X=[\alpha, \beta]+[\gamma, \delta]$, where $\alpha, \beta, \gamma, \delta$ are distinct vertices in $S$ such that $\alpha \beta, \gamma \delta, \alpha \gamma, \alpha \delta \in E$. Let $\mathcal{H}_{\mathbb{Q}}$ denote the quotient $\mathcal{N}_{\mathbb{Q}} /\langle X\rangle$, where $\langle X\rangle$ is the onedimensional subspace spanned by $X$. Let $\mathcal{H}=\mathcal{N} /\langle X\rangle$. It was proved in [5] that if the graph $(S, E)$ is a complete graph (i.e. $\alpha \beta \in E$ for all $\alpha, \beta \in S$ ), then $\mathcal{H}_{\mathbb{Q}}$ does not admit a hyperbolic automorphism whose characteristic polynomial has integer coefficients and unit constant term. (See Theorem 3.2 of [5].) We prove a similar result for an arbitrary graph.

THEOREM 5.1. The 2-step nilpotent Lie algebra $\mathcal{H}_{\mathbb{Q}}$, defined as above, does not admit a hyperbolic automorphism whose characteristic polynomial has integer coefficients and unit constant term.

Notation 5.2. We recall that $\mathcal{N}=V \oplus W$. (See $\S 3$.) We decompose $\mathcal{H}$ as $\mathcal{H}=V \oplus W^{\prime}$, where $W^{\prime}=W /\langle X\rangle$. Let $\bar{G}$ be the subgroup of $G L(V)$ consisting of 
all restrictions $\tau \mid V$ such that $\tau \in \operatorname{Aut}(\mathcal{H})$ and $\tau(V)=V$. Let $G$ be the subgroup of $G L(V)$ consisting of all restrictions $\tau \mid V$ such that $\tau \in \operatorname{Aut}(\mathcal{N})$ and $\tau(V)=V$. It can be seen that subgroups $G$ and $\bar{G}$ of $G L(V)$ are Lie subgroups. Let $\mathcal{G}$ (resp. $\overline{\mathcal{G}})$ be the Lie algebra of $G$ (resp. $\bar{G}$ ). Let $G^{0}$ (resp. $\bar{G}^{0}$ ) be the connected component of the identity in $G$ (resp. in $\bar{G}$ ). Let $\mathcal{D}$ (resp. $\overline{\mathcal{D}}$ ) be the Lie subalgebra of $\mathcal{G}$ (resp. of $\overline{\mathcal{G}})$ consisting of all endomorphisms in $\mathcal{G}$ (resp. in $\overline{\mathcal{G}}$ ) that are represented by diagonal matrices with respect to the basis $S$. Note that $\overline{\mathcal{D}}$ consists of all the endomorphisms in $\mathcal{D}$ that are contained in $\overline{\mathcal{G}}$. For $\eta, \zeta \in S$, let $E_{\eta \zeta}$ be the element of $\operatorname{End}(V)$ such that $E_{\eta \zeta}(\zeta)=\eta$ and $E_{\eta \zeta}(\xi)=0$ for all $\xi \in S, \xi \neq \zeta$.

Notation 5.3. Recall that $X=[\alpha, \beta]+[\gamma, \delta]$, where $\alpha, \beta, \gamma, \delta$ are distinct vertices in $S$ such that $\alpha \beta, \gamma \delta, \alpha \gamma, \alpha \delta \in E$. Let $S^{\prime}=\{\alpha, \beta, \gamma, \delta\}$. Let $W_{\phi \psi}^{\phi^{\prime} \psi^{\prime}}$ denote the subspace of $\operatorname{End}(V)$ spanned by $E_{\phi \psi}$ and $E_{\phi^{\prime} \psi^{\prime}}$, where $\left\{\phi, \psi, \phi^{\prime}, \psi^{\prime}\right\}=S^{\prime}$.

Proposition 5.4. The Lie algebra $\overline{\mathcal{G}}$, defined as above, is spanned by $\overline{\mathcal{D}}, W_{\alpha \delta}^{\gamma \beta} \cap \overline{\mathcal{G}}$, $W_{\beta \gamma}^{\delta \alpha} \cap \overline{\mathcal{G}}, W_{\alpha \gamma}^{\delta \beta} \cap \overline{\mathcal{G}}, W_{\gamma \alpha}^{\beta \delta} \cap \overline{\mathcal{G}}$, and the elements of $\overline{\mathcal{G}}$ of the following type: (i) $E_{\eta \zeta}$, where $\eta \neq \zeta, \eta, \zeta \notin S^{\prime}$, (ii) $E_{\eta \zeta}$, where $\eta \in S^{\prime}$ and $\zeta \notin S^{\prime}$, (iii) $E_{\eta \zeta}$, where $\eta \notin S^{\prime}$ and $\zeta \in S^{\prime}$, (iv) $E_{\alpha \beta}, E_{\gamma \delta}, E_{\beta \alpha}, E_{\delta \gamma}$.

Proof. Let $Y \in \overline{\mathcal{G}}$. Then $Y$ can be expressed as $Y=Y_{0}+\sum_{\eta, \zeta \in S, \eta \neq \zeta} a_{\eta \zeta} E_{\eta \zeta}$, where $Y_{0} \in \mathcal{D}$ (see Notation 5.2) and $a_{\eta \zeta} \in \mathbb{R}$. By using the fact that $E_{\zeta \zeta} \in \overline{\mathcal{G}}$ for all $\zeta \notin S^{\prime}$, we observe that $a_{\eta \zeta} E_{\eta \zeta}$ is contained in $\overline{\mathcal{G}}$ for all $\eta, \zeta \notin S^{\prime}$. (See the proof of Proposition 3.1 in [3].) We note that $E_{\alpha \alpha}+E_{\gamma \gamma}, E_{\beta \beta}+E_{\delta \delta}, E_{\beta \beta}+E_{\gamma \gamma}$ are contained in $\overline{\mathcal{G}}$. Since $\left[E_{\zeta \zeta},\left[E_{\alpha \alpha}+E_{\gamma \gamma}, Y\right]\right]$ and $\left[E_{\zeta \zeta},\left[E_{\zeta \zeta},\left[E_{\alpha \alpha}+E_{\gamma \gamma}, Y\right]\right]\right]$ are in $\overline{\mathcal{G}}$ for $\zeta \notin S^{\prime}, a_{\zeta \alpha} E_{\zeta \alpha}+$ $a_{\zeta \gamma} E_{\zeta \gamma}$ and $a_{\alpha \zeta} E_{\alpha \zeta}+a_{\gamma \zeta} E_{\gamma \zeta}$ are contained in $\overline{\mathcal{G}}$. Now as $E_{\alpha \alpha}+E_{\delta \delta} \in \overline{\mathcal{G}}$, we have $a_{\zeta \alpha} E_{\zeta \alpha}$ and $a_{\alpha \zeta} E_{\alpha \zeta}$ are in $\overline{\mathcal{G}}$. Similarly we can see that $a_{\eta \zeta} E_{\eta \zeta} \in \overline{\mathcal{G}}$, for all $\eta \in S^{\prime}$ and $\zeta \notin S^{\prime}$; also $a_{\eta \zeta} E_{\eta \zeta} \in \overline{\mathcal{G}}$, for all $\eta \notin S^{\prime}$ and $\zeta \in S^{\prime}$. We also have $\left[E_{\beta \beta}+E_{\gamma \gamma},\left[E_{\beta \beta}+\right.\right.$ $\left.\left.E_{\delta \delta},\left[E_{\alpha \alpha}+E_{\gamma \gamma}, Y\right]\right]\right] \in \overline{\mathcal{G}}$. This shows that $Z=a_{\gamma \delta} E_{\gamma \delta}+a_{\beta \alpha} E_{\beta \alpha}-a_{\delta \gamma} E_{\delta \gamma}-a_{\alpha \beta} E_{\alpha \beta} \in$ $\overline{\mathcal{G}}$. Also $\left[E_{\beta \beta}+E_{\gamma \gamma}, Z\right] \in \overline{\mathcal{G}}$. Therefore we get $a_{\gamma \delta} E_{\gamma \delta}+a_{\beta \alpha} E_{\beta \alpha}+a_{\delta \gamma} E_{\delta \gamma}+a_{\alpha \beta} E_{\alpha \beta} \in$ $\overline{\mathcal{G}}$. Hence $a_{\gamma \delta} E_{\gamma \delta}+a_{\beta \alpha} E_{\beta \alpha}$ and $a_{\delta \gamma} E_{\delta \gamma}+a_{\alpha \beta} E_{\alpha \beta}$ are contained in $\overline{\mathcal{G}}$. Since $\left[E_{\alpha \alpha}+\right.$ $\left.E_{\gamma \gamma}, a_{\gamma \delta} E_{\gamma \delta}+a_{\beta \alpha} E_{\beta \alpha}\right] \in \overline{\mathcal{G}}$, we have $a_{\gamma \delta} E_{\gamma \delta}-a_{\beta \alpha} E_{\beta \alpha} \in \overline{\mathcal{G}}$ and so $a_{\gamma \delta} E_{\gamma \delta} \in \overline{\mathcal{G}}$. Hence we have proved that if $a_{\gamma \delta} \neq 0$ then $E_{\gamma \delta} \in \overline{\mathcal{G}}$. Similarly it can be proved that $E_{\beta \alpha} \in \overline{\mathcal{G}}$ if $a_{\beta \alpha} \neq 0, E_{\alpha \beta} \in \overline{\mathcal{G}}$ if $a_{\alpha \beta} \neq 0$, and $E_{\delta \gamma} \in \overline{\mathcal{G}}$ if $a_{\delta \gamma} \neq 0$. Now $Z^{\prime}=\left[E_{\alpha \alpha}+E_{\gamma \gamma},\left[E_{\beta \beta}+\right.\right.$ $\left.\left.E_{\delta \delta},\left[E_{\alpha \alpha}+E_{\gamma \gamma}, Y\right]\right]\right] \in \overline{\mathcal{G}}$, and hence

$$
\left[E_{\beta \beta}+E_{\delta \delta},\left[E_{\alpha \alpha}+E_{\gamma \gamma}, Y\right]\right]+Z^{\prime} \in \overline{\mathcal{G}} .
$$

As $a_{\alpha \beta} E_{\alpha \beta}$ and $a_{\gamma \delta} E_{\gamma \delta}$ are contained in $\overline{\mathcal{G}}$, we have $a_{\alpha \delta} E_{\alpha \delta}+a_{\gamma \beta} E_{\gamma \beta} \in \overline{\mathcal{G}}$. Similarly we can prove that $a_{\beta \gamma} E_{\beta \gamma}+a_{\delta \alpha} E_{\delta \alpha} \in \overline{\mathcal{G}}$. As $Y \in \overline{\mathcal{G}}$, by the observations above we have $Z^{\prime \prime}=Y_{0}+a_{\alpha \gamma} E_{\alpha \gamma}+a_{\gamma \alpha} E_{\gamma \alpha}+a_{\beta \delta} E_{\beta \delta}+a_{\delta \beta} E_{\delta \beta} \in \overline{\mathcal{G}}$. Considering the element $\left[E_{\alpha \alpha}+\right.$ $\left.E_{\delta \delta}, Z^{\prime \prime}\right]$ we prove that $a_{\alpha \gamma} E_{\alpha \gamma}+a_{\delta \beta} E_{\delta \beta} \in \overline{\mathcal{G}}$ and $a_{\gamma \alpha} E_{\gamma \alpha}+a_{\beta \delta} E_{\beta \delta}$ are in $\overline{\mathcal{G}}$. Hence we have now $Y_{0} \in \overline{\mathcal{G}}$. As $Y_{0}$ is in $\mathcal{D}, Y_{0} \in \overline{\mathcal{D}}$. Hence we have proved our claim that $\overline{\mathcal{G}}$ is spanned by $\overline{\mathcal{D}}, W_{\alpha \delta}^{\gamma \beta} \cap \overline{\mathcal{G}}, W_{\beta \gamma}^{\delta \alpha} \cap \overline{\mathcal{G}}, W_{\alpha \gamma}^{\delta \beta} \cap \overline{\mathcal{G}}, W_{\gamma \alpha}^{\beta \delta} \cap \overline{\mathcal{G}}$, and the elements of $\overline{\mathcal{G}}$ of the type (i)-(iv) as stated.

Proposition 5.5. Any automorphism $\bar{T}$ in $\bar{G}^{0}$ is induced by an automorphism $T$ in $G^{0}$, with $T(\langle X\rangle)=\langle X\rangle$.

Proof. We shall prove the following results. If the element from the type (i)-(iv) in the statement of Proposition 5.4, considered as an element of $\operatorname{End}(V)$, is in the Lie algebra $\overline{\mathcal{G}}$, then that element is in $\mathcal{G}$. (See Notation 5.2.) We shall also prove that 
$W_{\alpha \delta}^{\gamma \beta} \cap \overline{\mathcal{G}}, W_{\beta \gamma}^{\delta \alpha} \cap \overline{\mathcal{G}}, W_{\alpha \gamma}^{\delta \beta} \cap \overline{\mathcal{G}}$, and $W_{\gamma \alpha}^{\beta \delta} \cap \overline{\mathcal{G}}$ are contained in $\mathcal{G}$. Let $I$ denote the identity transformation in $G L(V)$.

(i) If $E_{\eta \zeta} \in \overline{\mathcal{G}}$, where $\eta \neq \zeta, \eta, \zeta \notin S^{\prime}$, then $\tau=I+E_{\eta \zeta} \in \bar{G}$. We shall prove that $\Omega^{\prime}(\eta) \subset \Omega(\zeta)$. (See $\S 4$.) Suppose that $\xi \notin \Omega(\zeta)$. Then we have $[\xi, \zeta]=0$. As $\tau \in \bar{G}$, it is the restriction of a Lie automorphisms of $\mathcal{H}$, and hence we get that $[\tau(\xi), \tau(\zeta)]=0$ in $\mathcal{H}$. Therefore we have $[\xi, \zeta+\eta]=c([\alpha, \beta]+[\gamma, \delta])$, where $c \in \mathbb{R}$. As $[\xi, \zeta]=0$, we have $[\xi, \eta]=c([\alpha, \beta]+[\gamma, \delta])$ and so $c=0$. Hence $\xi \notin \Omega^{\prime}(\eta)$. This shows that $\Omega^{\prime}(\eta) \subset \Omega(\zeta)$, and so $E_{\eta \zeta} \in \mathcal{G}$, where $\eta \neq \zeta, \eta, \zeta \notin S^{\prime}$. (See Proposition 4.1 in [3].)

(ii) Consider the element $E_{\eta \alpha}$, where $\eta \notin S^{\prime}$. Suppose that $E_{\eta \alpha} \in \overline{\mathcal{G}}$ Let $\tau=I+$ $E_{\eta \alpha}$. If $\zeta \notin \Omega(\alpha)$, then $[\zeta, \alpha]=0$. As $\tau \in \overline{\mathcal{G}}$, we have $[\zeta, \alpha+\eta]=0$ in $\mathcal{H}$. By the same argument as above we have $[\zeta, \eta]=0$. Hence $\Omega^{\prime}(\eta) \subset \Omega(\alpha)$, so that $E_{\eta \alpha} \in \mathcal{G}$.

By similar arguments we can prove our claim for the elements of the type (ii), (iii) and (iv).

We shall prove that any element of $W_{\alpha \delta}^{\gamma \beta} \cap \overline{\mathcal{G}}$, considered as an element of $\operatorname{End}(V)$, is contained in $\mathcal{G}$. Suppose now that the linear combination of $E_{\alpha \delta}$ and $E_{\gamma \beta}$, say $a E_{\alpha \delta}+$ $b E_{\gamma \beta}$, is in $\overline{\mathcal{G}}$. Then $\tau=I+t\left(E_{\alpha \delta}+b E_{\gamma \beta}\right) \in \bar{G}, t \neq 0$. We show that the subspace of $\wedge^{2} V, W^{\prime}$ say, spanned by the set of all $\zeta \wedge \eta$ such that $\zeta \eta$ is not an edge, is $\wedge^{2} \tau$-invariant. Let $\zeta, \eta \in S$ such that $\zeta \neq \eta$ and $\zeta \eta$ is not an edge. If neither of $\zeta$ and $\eta$ is contained in $\{\delta, \beta\}$, then $\wedge^{2} \tau(\zeta \wedge \eta)=\zeta \wedge \eta$. If $\zeta=\delta$ and $\eta \neq \beta$, then as $\tau \in \bar{G}$, we have $\tau[\zeta, \eta]=0$ in $\mathcal{H}$, and hence $[\operatorname{ta} \alpha+\delta, \eta]=c([\alpha, \beta]+[\gamma, \delta])$ in $\mathcal{N}$, where $c \in \mathbb{R}$. Therefore either $a=0$ or $\alpha \eta$ is not an edge. In both the cases we have $\wedge^{2} \tau(\zeta \wedge \eta) \in W^{\prime}$. Similarly if $\zeta=\beta$ and $\eta \neq \delta$ we are through. Now if $\zeta=\delta$ and $\eta=\beta$, we have $\tau[\delta, \beta]=0$ in $\mathcal{H}$. Hence $[\operatorname{ta} \alpha+\delta, t b \gamma+\beta]=c([\alpha, \beta]+[\gamma, \delta])$ in $\mathcal{N}$, where $c \in \mathbb{R}$. As $\delta \beta$ is not an edge and $\alpha \gamma$ is an edge, we have $t^{2} a b[\alpha, \gamma]+t a[\alpha, \beta]+t b[\delta, \gamma]=c([\alpha, \beta]+[\gamma, \delta])$ in $\mathcal{N}$, and hence $a b=0$ and $a=-b$. Therefore $\tau=I$. Thus we have proved that $W^{\prime}$ is $\wedge^{2} \tau$-invariant. Therefore $\tau \in G$, and hence $a E_{\alpha \delta}+b E_{\gamma \beta} \in \mathcal{G}$.

Similarly we see that our claim holds for the elements of $W_{\beta \gamma}^{\delta \alpha} \cap \overline{\mathcal{G}}, W_{\alpha \gamma}^{\delta \beta} \cap \overline{\mathcal{G}}$, and $W_{\gamma \alpha}^{\beta \delta} \cap \overline{\mathcal{G}}$.

By using the argument above, Proposition 5.4 and Theorem 2.10.1 in [11], we see that there exists an open neighbourhood $U$ of $I$ in $\bar{G}^{0}$ such that any automorphism contained in $U$ can be lifted to an automorphism of $\mathcal{N}$ that keeps an ideal $\langle X\rangle$ invariant. Hence any automorphism $\bar{T}$ in $\bar{G}^{0}$ can be lifted to an automorphism $T$ in $G^{0}$ such that $T(\langle X\rangle)=\langle X\rangle$. (Use Proposition 3.18 in [12].)

Proof of Theorem 5.1. Suppose $\bar{\theta} \in \operatorname{Aut}\left(\mathcal{H}_{\mathbb{Q}}\right)$ is a hyperbolic automorphism such that its characteristic polynomial has integer coefficients and unit constant term. Since $\operatorname{Aut}(\mathcal{H})$ has finitely many connected components, by replacing $\bar{\theta}$ by its suitable power we may assume that $\bar{\theta}$ is contained in the connected component of the identity in $\operatorname{Aut}(\mathcal{H})$. By Proposition 5.5, we see that there exists an automorphism $\theta$ contained in the connected component of the identity of $\operatorname{Aut}(\mathcal{N})$ such that its characteristic polynomial has integer coefficients and unit constant, $\theta(X)=X$, and $\theta$ has an eigenvalue 1 of multiplicity 1 . We can assume that the matrix of $\theta$ with respect to the basis $S \cup E$ is an integer matrix.

We have $\theta\left(\mathcal{N}_{j}\right)=\mathcal{N}_{j}$, for each $j=1, \ldots, k$, where $\mathcal{N}_{j}=\left(\oplus_{i \leq j} V_{\lambda_{i}}\right) \oplus W$ and $\lambda_{1}, \ldots, \lambda_{k}$ is an enumeration of $\Lambda$ such that for all $j=1, \ldots, m, \bigoplus_{i \leq j} V_{\lambda_{i}}$ is invariant under the action of $G^{0}$. (See $\S 2$.) Let $\pi_{j}: \mathcal{N}_{j} \rightarrow V_{\lambda_{j}}$ denote the canonical projection for each $j=1, \ldots, k$. Let $\theta_{\lambda_{j}}: V_{\lambda_{j}} \rightarrow V_{\lambda_{j}}$ be given by $\theta_{\lambda_{j}}=\pi_{j} \circ \theta$.

We have $W=\sum_{\lambda, \mu \in \Lambda}\left[V_{\lambda}, V_{\mu}\right]$. All the eigenvalues of $\theta$ on $W$ are pairwise products of the eigenvalues on $V_{\lambda}$ 's. Also $a_{\lambda} a_{\mu}$ occurs as an eigenvalue of $\theta \mid W$ if and only if 
there exists $\zeta \in S_{\lambda}$ and $\eta \in S_{\mu}$ (notation is as before) such that $\zeta \eta$ is an edge, where $a_{\lambda}$ is an eigenvalue of $\theta_{\lambda}$ and $a_{\mu}$ is an eigenvalue of $\theta_{\mu}$ As 1 is an eigenvalue of $\theta$, there exist $\lambda$ and $\mu$ in $\Lambda$ such that $\zeta \eta$ is an edge for $\zeta \in S_{\lambda}$ and $\eta \in S_{\mu}$, and $a_{\lambda} a_{\mu}=1, a_{\lambda}$ and $a_{\mu}$ being eigenvalues of $\theta_{\lambda}$ and $\theta_{\mu}$ respectively.

We shall prove that $\lambda=\mu$. Suppose that $\lambda \neq \mu$. Let $a_{\lambda}^{\prime}$ be a conjugate of $a_{\lambda}$ over $\mathbb{Q}$, $a_{\lambda}^{\prime} \neq a_{\lambda}$. Then $a_{\mu}=a_{\lambda}^{-1}$ and $a_{\lambda^{\prime}}^{-1}$ are conjugates over $\mathbb{Q}$. The minimal polynomial of $a_{\mu}$ over $\mathbb{Q}$ divides the characteristic polynomial of $\theta_{\mu}$. Hence $a_{\lambda^{\prime}}^{-1}$ occurs as an eigenvalue of $\theta_{\mu}$. As, by our assumption, $\zeta \eta$ is an edge for all $\zeta \in S_{\lambda}$ and $\eta \in S_{\mu}, a_{\lambda^{\prime}} a_{\lambda^{\prime}}^{-1}$ occurs as an eigenvalue of $\theta$, where $a_{\lambda} \neq a_{\lambda}^{\prime}$, and hence we arrive at a contradiction, as the multiplicity of the eigenvalue 1 is 1 . Therefore $\lambda=\mu$. Hence there exists $\lambda \in \Lambda$ such that the restriction of a graph $(S, E)$ on $S_{\lambda}$ is complete and $a_{\lambda} a_{\lambda}^{\prime}=1$ for the same eigenvalues $a_{\lambda}$ and $a_{\lambda}^{\prime}$ of $\theta_{\lambda}$, and $X \in\left[V_{\lambda}, V_{\lambda}\right]$, which is not possible (by Theorem 3.2 of [4]). This completes the proof of the theorem.

REMARK 5.6. Let $H$ be the simply connected nilpotent Lie group corresponding to the Lie algebra $\mathcal{H}$. Let $\Gamma$ be a lattice in $H$ corresponding to $\mathcal{H}_{\mathbb{Q}}$. (See [4].) Then Theorem 5.1 shows that the nilmanifold $\Gamma \backslash H$ does not admit an Anosov automorphism.

6. Nonexistence of Anosov automorphisms on 3-step nilmanifolds. In this section we study quotients of certain 3-step nilpotent Lie algebras. Let $(S, E)$ be a graph. Let $V$ be a vector space over $\mathbb{Q}$ with a basis as $S$. Let $\mathcal{F}_{3}(V)$ denote the free 3-step nilpotent Lie algebra over the rationals on $V$. Let $\mathcal{Q}=\mathcal{F}_{3}(V) / \mathcal{I}$. Here $\mathcal{I}$ is an ideal of $\mathcal{F}_{3}(V)$ generated by the elements $[\alpha, \beta]$, where $\alpha, \beta \in S$ and $\alpha \beta$ is not an edge. We decompose $\mathcal{Q}$ as $\mathcal{Q}=V \oplus W \oplus V_{3}$, where $V_{3}$ is the space spanned by all $[\alpha,[\beta, \gamma]]$ such that $\alpha, \beta, \gamma \in S$, and $\beta \gamma$ is an edge. Let $X$ be a nonzero vector in $V_{3}$. Let $\mathcal{M}=\mathcal{Q} /\langle X\rangle$, where $\langle X\rangle$ denotes the ideal generated by $X$ in $\mathcal{Q}$ which is a one-dimensional subspace spanned by $X$ in $\mathcal{Q}$.

Proposition 6.1. Let $\bar{\theta}$ be an automorphism of $\mathcal{M}$. Then there exists $\theta$, an automorphism of $\mathcal{Q}$ such that $\theta(X)=c X$, where $c$ is a nonzero rational. Furthermore $\theta$ induces an automorphism $\theta^{\prime}$ of $\mathcal{M}$ such that both $\bar{\theta}$ and $\theta^{\prime}$ have the same eigenvalues; also we have $\theta(V)=V, \theta(W)=W$ and $\theta\left(V_{3}\right)=V_{3}$.

Proof. Consider the linear endomorphism $T$ of $V$ defined by $T(\alpha)=\pi(\bar{\theta}(\bar{\alpha}))$ for all $\alpha \in S$, where $\pi$ is a natural projection of $\mathcal{M}$ onto $V$ with respect to the decomposition of $\mathcal{M}$ as $\mathcal{M}=V \oplus W \oplus V_{3} /\langle X\rangle$, and $\bar{\alpha}$ denotes the coset in $\mathcal{M}$ represented by $\alpha$. Note that $T$ is an automorphism of $V$. For, if $T(v)=0, v \in V$, then $\bar{\theta}(v) \in[\mathcal{M}, \mathcal{M}]$. But as $\bar{\theta}$ is an automorphism of $\mathcal{M}$, we must have $v=0$.

We then have a Lie algebra automorphism $\bar{T}$ of $\mathcal{F}_{3}(V)$ such that $\left.\bar{T}\right|_{V}=T$. We prove that $\bar{T}(\mathcal{I})=\mathcal{I}$. Suppose $\alpha, \beta$ are in $S$ such that $\alpha \beta$ is not an edge. By definition, $\bar{T}[\alpha, \beta]=[\pi(\bar{\theta}(\bar{\alpha})), \pi(\bar{\theta}(\bar{\beta}))]$. Now as $\alpha \beta$ is not an edge, we have $[\bar{\alpha}, \bar{\beta}]=0$. Hence $[\bar{\theta}(\bar{\alpha}), \bar{\theta}(\bar{\beta})]=0$ in $\mathcal{M}$. This implies that $[\pi(\bar{\theta}(\bar{\alpha})), \pi(\bar{\theta}(\bar{\beta}))] \in V_{3}$. Hence we have $[\pi(\bar{\theta}(\bar{\alpha})), \pi(\bar{\theta}(\bar{\beta}))]=0$ in $\mathcal{Q}$. Hence in $\mathcal{F}_{3}(V)$, we have $[\pi(\bar{\theta}(\bar{\alpha})), \pi(\bar{\theta}(\bar{\beta}))] \in \mathcal{I}$. Thus we have proved that $\bar{T}(\mathcal{I})=\mathcal{I}$ and hence $\bar{T}$ factors through an automorphism of $\mathcal{Q}$. Let $\theta$ denote the automorphism of $\mathcal{Q}$ induced by $\bar{T}$.

We claim that $\theta(X) \in\langle X\rangle$. We note that $\bar{\theta}(\bar{Y})=\overline{\theta(Y)}$ in $\mathcal{M}$, for all $Y \in V_{3}$, where bar is taken to denote the elements in $\mathcal{M}$ represented by elements in $\mathcal{Q}$. Now $\bar{\theta}(\bar{X})=\overline{0}$ in $\mathcal{M}$, as $\bar{\theta}$ is an automorphism of $\mathcal{M}$. This implies that $\theta(X) \in\langle X\rangle$ in $\mathcal{Q}$. Hence we have $\theta(X)=c X$, where $c$ is a nonzero rational. By definition of $\theta, \theta(V)=V, \theta(W)=W$, 
and $\theta\left(V_{3}\right)=V_{3}$. Let $\theta^{\prime}$ be the automorphism of $\mathcal{M}$ induced by $\theta$. Then both $\bar{\theta}$ and $\theta^{\prime}$ induce the same linear endomorphism on $\mathcal{M} /[\mathcal{M}, \mathcal{M}]$. Hence $\bar{\theta}$ and $\theta^{\prime}$ have same eigenvalues.

NotATION 6.2. Let $\left\{S_{\lambda}\right\}_{\lambda \in \Lambda}$ denote the family of coherent components (see $\S 2$ ) of the graph $(S, E)$. Let $\mathcal{E}$ be the set of all unordered pairs $\lambda \mu$ with $\lambda, \mu \in \Lambda$, such that $\alpha \beta \in E$, for $\alpha \in S_{\lambda}$ and $\beta \in S_{\mu}$. We recall that $V_{\lambda}$ denotes the subspace of $V$ spanned by $S_{\lambda}(\lambda \in \Lambda)$. Let $\lambda_{1}, \ldots, \lambda_{k}$ be an enumeration of $\Lambda$ such that assertion (ii) of Theorem 2.1 holds. Let $\mathcal{N}_{j}=\left(\oplus_{i \leq j} V_{\lambda_{i}}\right) \oplus W$ for $j=1, \ldots, k$.

THEOREM 6.3. The Lie algebra $\mathcal{M}$ does not admit a hyperbolic automorphism whose characteristic polynomial has integer coefficients and unit constant term.

Proof. Let $\bar{\theta}$ be a hyperbolic automorphism of $\mathcal{M}$ such that the characteristic polynomial of $\bar{\theta}$ has integer coefficients and unit constant term. Let $\theta$ be an automorphism of $\mathcal{Q}$ as obtained in the previous proposition. Let $\tau$ be an automorphism of $\mathcal{N}$ induced by $\theta$. As $\bar{\theta}$ is a hyperbolic automorphism, and $\theta(X)=X, 1$ is an eigenvalue of $\theta$ of multiplicity 1 . Since the characteristic polynomial of $\bar{\theta}$ has integer coefficients and unit constant term, we may assume that the matrix of $\theta$ has all integer entries (by replacing $\theta$ by some power of $\theta$ if necessary.) As $\operatorname{Aut}(\mathcal{N})$ has finitely many components, we may assume that $\theta$ lies in the connected component of the identity of $\operatorname{Aut}(\mathcal{Q})$ and $\tau$ lies in the connected component of the identity of $\operatorname{Aut}(\mathcal{N})$. Hence $\theta\left(\mathcal{N}_{j}\right)=\mathcal{N}_{j}$. (See Lemma 2.2.)

Let $\pi_{\lambda_{j}}: \mathcal{N}_{j} \rightarrow V_{\lambda_{j}}$ denote the canonical projection. Let $\theta_{\lambda_{j}}$ be an endomorphism of $V_{\lambda_{j}}$ defined by $\theta_{\lambda_{j}}(v)=\pi_{\lambda_{j}}(\theta(v))$, for all $v \in V_{\lambda_{j}}$. We note that $\theta_{\lambda_{j}}$ is an automorphism of $V_{\lambda_{j}}$.

All the eigenvalues of $\theta$ restricted on $V_{3}$ are of the following type:

(i) $\delta_{\lambda} \delta_{\mu} \delta_{\nu}$, where $\delta_{\lambda}, \delta_{\mu}$, and $\delta_{v}$ are the eigenvalues of $\theta_{\lambda}, \theta_{\mu}$, and $\theta_{v}$ respectively and $\mu \nu \in \mathcal{E}$; see Notation 6.2.

(ii) $\delta_{\lambda} \delta_{\lambda}^{\prime} \delta_{\lambda}^{\prime \prime}$, where $\delta_{\lambda}, \delta_{\lambda}^{\prime}, \delta_{\lambda}^{\prime \prime}$ are the eigenvalues of $\theta_{\lambda}$ and the restriction of $(S, E)$ to $S_{\lambda}$ is a complete graph.

As $\left.\theta\right|_{V_{3}}$ has an eigenvalue 1, we have the following two cases.

Case (i). Suppose $\delta_{\lambda} \delta_{\mu} \delta_{\nu}=1$, where $\delta_{\lambda}, \delta_{\mu}$, and $\delta_{\nu}$ are the eigenvalues of $\theta_{\lambda}, \theta_{\mu}$, and $\theta_{\nu}$ respectively, and $\mu \nu \in \mathcal{E}$. Now as $\mu \nu \in \mathcal{E}, \delta_{\mu} \delta_{\nu}$ occurs as an eigenvalue of $\left.\theta\right|_{W}$. Thus we have an invertible matrix, say $A$, with integer entries such that $\delta_{\mu} \delta_{\nu}$ is an eigenvalue of $A$. Also we have an invertible matrix, say $B$, with integer entries having $\delta_{\lambda}=\left(\delta_{\mu} \delta_{\nu}\right)^{-1}$ as an eigenvalue. Hence there exists an eigenvalue of the type $\delta_{\mu}^{\prime} \delta_{v}^{\prime}$ of $A$ and $\delta_{\lambda}^{\prime}=\left(\delta_{\mu}^{\prime} \delta_{v}^{\prime}\right)^{-1}$ of $B$ such that $\delta_{\mu}^{\prime}, \delta_{v}^{\prime}$ and $\delta_{\lambda}^{\prime}$ are the eigenvalues of $\theta_{\mu}, \theta_{v}$ and $\theta_{\lambda}$ respectively, and $\delta_{\mu}^{\prime} \delta_{\nu}^{\prime} \neq \delta_{\mu} \delta_{\nu}$. This contradicts the fact that the multiplicity of the eigenvalue 1 is 1 .

Case (ii). Suppose $\delta_{\lambda} \delta_{\lambda}^{\prime} \delta_{\lambda}^{\prime \prime}=1$ for some $\lambda \in \Lambda$ such that the restriction of $(S, E)$ on $S_{\lambda}$ is complete and $\delta_{\lambda}, \delta_{\lambda}^{\prime}, \delta_{\lambda}^{\prime \prime}$ are the eigenvalues of $\theta_{\lambda}$. If $\delta_{\lambda}=\delta_{\lambda}^{\prime}=\delta_{\lambda}^{\prime \prime}$, then $\delta_{\lambda}^{3}=1$, which is a contradiction, since $\bar{\theta}$ is hyperbolic. Hence we may assume that $\delta_{\lambda}^{\prime} \neq \delta_{\lambda}^{\prime \prime}$. Let $V_{\lambda}^{\mathbb{C}}$ denote the complexification of $V_{\lambda}$. Suppose that $Y, Y^{\prime}, Y^{\prime \prime} \in V_{\lambda}^{\mathbb{C}}$ are eigenvectors corresponding to the eigenvalues $\delta_{\lambda}, \delta_{\lambda}^{\prime}, \delta_{\lambda}^{\prime \prime}$. We consider the complexification of $\mathcal{Q}$ and $\theta$ also. As $\delta_{\lambda}^{\prime} \neq \delta_{\lambda}^{\prime \prime}, Y^{\prime}$ and $Y^{\prime \prime}$ are linearly independent. Hence $\left[Y,\left[Y^{\prime}, Y^{\prime \prime}\right]\right] \neq 0$ in $\mathcal{Q}$ and also we have $\theta\left[\left[Y,\left[Y^{\prime}, Y^{\prime \prime}\right]\right]=\left[Y,\left[Y^{\prime}, Y^{\prime \prime}\right]\right]\right.$. If all the $\delta_{\lambda}, \delta_{\lambda}^{\prime}, \delta_{\lambda}^{\prime \prime}$ are distinct, then consider $\left[Y^{\prime},\left[Y, Y^{\prime \prime}\right]\right]$, which is an eigenvector corresponding to the eigenvalue 1 . Also $\left[Y^{\prime},\left[Y, Y^{\prime \prime}\right]\right]$ and $\left[Y,\left[Y^{\prime}, Y^{\prime \prime}\right]\right]$ are linearly independent. This is not possible as 
the eigenvalue 1 has multiplicity 1 . If $\delta_{\lambda}=\delta_{\lambda}^{\prime \prime}$ and $Z \in W^{\mathbb{C}}$ is an eigenvector of $\theta$ corresponding to the eigenvalue $\delta_{\lambda}^{2}$, then consider $\left[Y^{\prime}, Z\right]$, which is an eigenvector of $\theta$ corresponding to the eigenvalue 1 . Also $\left[Y^{\prime}, Z\right]$ and $\left[Y,\left[Y^{\prime}, Y^{\prime \prime}\right]\right.$ are linearly independent. This is a contradiction. Similarly we get a contradiction if $\delta_{\lambda}=\delta_{\lambda}^{\prime}$. This proves the theorem.

REMARK 6.4. Theorem 6.3 shows that the nilmanifold $\Gamma \backslash M$, where $M$ is the simply connected nilpotent Lie group corresponding to the Lie algebra $\mathcal{M} \otimes \mathbb{R}$ and $\Gamma$ is a lattice in $M$ corresponding to $\mathcal{M}$, does not admit an Anosov automorphism. In particular, a nilmanifold $\Gamma \backslash M$, where $\Gamma$ corresponds to the rational Lie algebra given by a quotient of free 3-step nilpotent Lie algebra by a one-dimensional ideal, does not admit an Anosov automorphism.

ACKNowledgements. I am very grateful to Professor S. G. Dani for his valuable help. I express my gratitude to Professor J. Lauret for his helpful comments and suggestions. I would like to thank TWAS, Trieste-Italy and CIEM, National University of Cordoba, Argentina for their support.

\section{REFERENCES}

1. L. Auslander and J. Scheuneman, On certain automorphisms of nilpotent Lie groups, in Global analysis (Proc. Sympos. Pure Math., Vol. XIV, Berkeley, Calif. 1968) (Amer. Math. Soc., Providence, 1970), 9-15.

2. S. G. Dani, Nilmanifolds with Anosov automorphisms, J. London Math. Soc. (2) 18 (1978), 553-559.

3. S. G. Dani and M. G. Mainkar, Anosov automorphisms on compact nilmanifolds associated with graphs, Trans. Amer. Math. Soc. 357 (2005), 2235-2251.

4. K. Dekimpe, Hyperbolic automorphisms and Anosov diffeomorphisms on nilmanifolds, Trans. Amer. Math. Soc. 353 (2001), 2859-2877.

5. K. Dekimpe and S. Deschamps, Anosov diffeomorphisms on a class of 2-step nilmanifolds, Glasgow Math. J. 45 (2003), 269-280.

6. J. Lauret, Examples of Anosov diffeomorphisms, J. Algebra 262 (2003), 201-209. Corrigendum: 268 (2003), 371-372.

7. J. Lauret and C. Will, Anosov diffeomorphisms on nilmanifolds up to dimension 8, appeared in http://arXiv.org/abs/math/0406199

8. A. Manning, There are no new Anosov diffeomorphisms on tori, Amer. J. Math 96 (1974), 422-429.

9. M. S. Raghunathan, Discrete subgroups of Lie groups (Springer-Verlag, 1972).

10. S. Smale, Differential dynamical systems, Bull. Amer. Math. Soc. 73 (1967), 747-817.

11. V. S. Varadarajan, Lie groups, Lie algebras and their representations, Graduate Texts in Mathematics No. 102 (Springer Verlag, 1984). (Reprinted edition).

12. Frank W. Warner, Foundations of differentiable manifolds and Lie groups (SpringerVerlag, 1983). 